\title{
Imatinib in PDGFR positive malignant solitary fibrous tumour of the pleura
}

\author{
Sarah Bigeni' ${ }^{1}$, Cynthia Jones ${ }^{1}$, Joseph Galea ${ }^{2}$ \\ ${ }^{1}$ Department of Acute Medicine, Mater Dei Hospital, Msida, Malta \\ ${ }^{2}$ Department of Cardiothoracic Surgery, Mater Dei Hospital, Msida, Malta
}

Received October 27, 2015; Revised February 07, 2016; Accepted February 09, 2016; Published Online February 15, 2016

Case Report

\begin{abstract}
In 2013, two cases of malignant solitary fibrous tumours (MSFTs) were diagnosed in Malta. Both were inoperable at presentation. Both tumours expressed platelet-derived growth factor receptor (PDGFR) with no mutations and the tyrosine kinase inhibitor imatinib was prescribed. Nonetheless, treatment was not as effective as documented in previous case-reports.
\end{abstract}

Keywords: Malignant Solitary Fibrous Tumours; Imatinib; Pleura; PDGFR

\section{Introduction}

First described in 1931 by Klemperer and Rabin, solitary fibrous tumour of the pleura (SFTP) is a mesenchymal neoplasm that usually involves the pleura. ${ }^{1}$ SFTP is more common from the fourth till the sixth decades of life and occurs with equal frequency in both genders. Only about 800 cases of solitary fibrous pleural tumours have been reported in the medical literature. ${ }^{2}$ The majority of these are benign (80\%) however rare cases of malignancy have been reported ${ }^{3}$.

It can occur in other thoracic areas (pericardium, lung and mediastinum) as well as in extra-thoracic areas (salivary glands, epiglottis, meninx, thyroid, breast and kidneys). ${ }^{5,6}$ In over half of the patients the tumour is asymptomatic, but the patient may present with cough, chest pain and dyspnoea. Complete en bloc surgical resection is the treatment of choice. ${ }^{3}$

Biologically MSFT of the pleura behave unpredictably. These also have a low sensitivity to conventional chemotherapy.7,8 A previous case-report showed evidence of the activity of imatinib in a symptomatic patient with a chemo- and radio-resistant advanced MSFT with platelet-derived growth factor receptor (PDGFR) expression, who survived 21-months and showed reduction in tumour metabolism ${ }^{6}$. It was suggested that the tyrosine kinase inhibitor imatinib should be considered for patients with recurrent or unresectable MSFTs with PDGFR expression 7 . There is no data available that the absence of PDGRF expression of the tumour leads to resistance of the tumour.

\section{Cases Presentation}

\subsection{Case 1}

A 67-year-old gentleman was referred to casualty for further investigation and treatment of dyspnoea and dry cough worse on lying down. He was an ex-smoker, hypertensive and used to work as a groundsman but had no previous exposure to asbestos. Three months previously the patient was reviewed by his GP and a chest X-ray (CXR) showed a small left-sided pleural effusion (Figure 1). During that occasion, the patient was not investigated further. However on this current admission a CXR at casualty showed a large left-sided pleural effusion (Figure 2).

A computed tomography (CT) of the thorax was done which confirmed the left-sided pleural effusion with collapse of the lower lobe of the ipsilateral lung. A huge mass with calcifications and smaller satellite masses were detected in the left hemithorax (Figure 3). A chest drain was inserted and after drainage of the pleural fluid, a large mass was revealed (Figure 4).

Pleural fluid was sent for cytology on two separate occasions both reporting absence of malignant cells.

Corresponding author: Joseph Galea; Department of Cardiothoracic Surgery, Mater Dei Hospital, Msida, Malta.

Cite this article as: Bigeni S, Jones C, Galea J. Imatinib in PDGFR positive malignant solitary fibrous tumour of the pleura. Int J Cancer Ther Oncol. 2016; 4(1):4116. DOI: 10.14319/ijcto.41.16

(A part of this research was presented at European Respiratory Society Congress, which was held from September 26-29, 2015 in Amsterdam, Netherlands.) 
Bronchoscopy was carried out and bronchial brushing and lavage did not reveal any malignant cells.

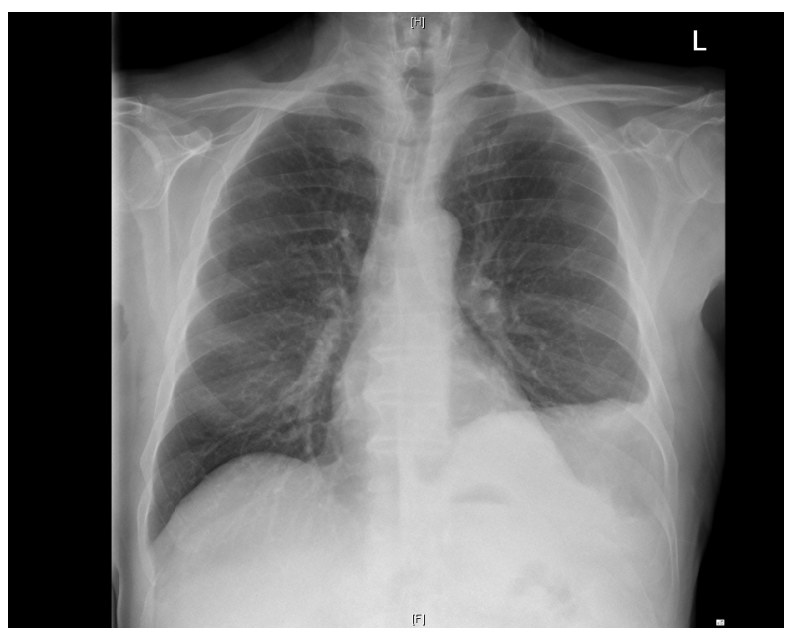

Figure 1: CXR done by GP three months prior to presentation at casualty.

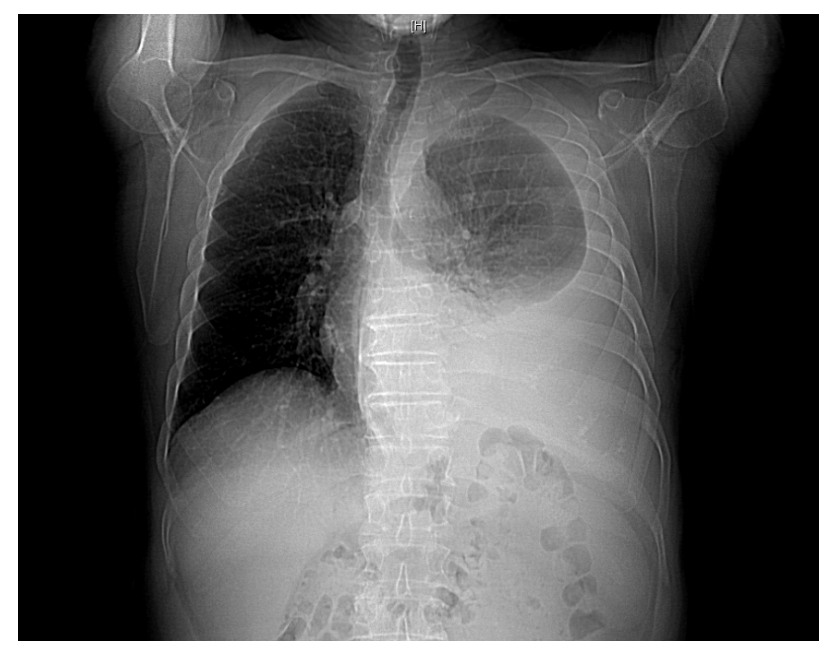

Figure 2: Medical imaging done at casualty on presentation.

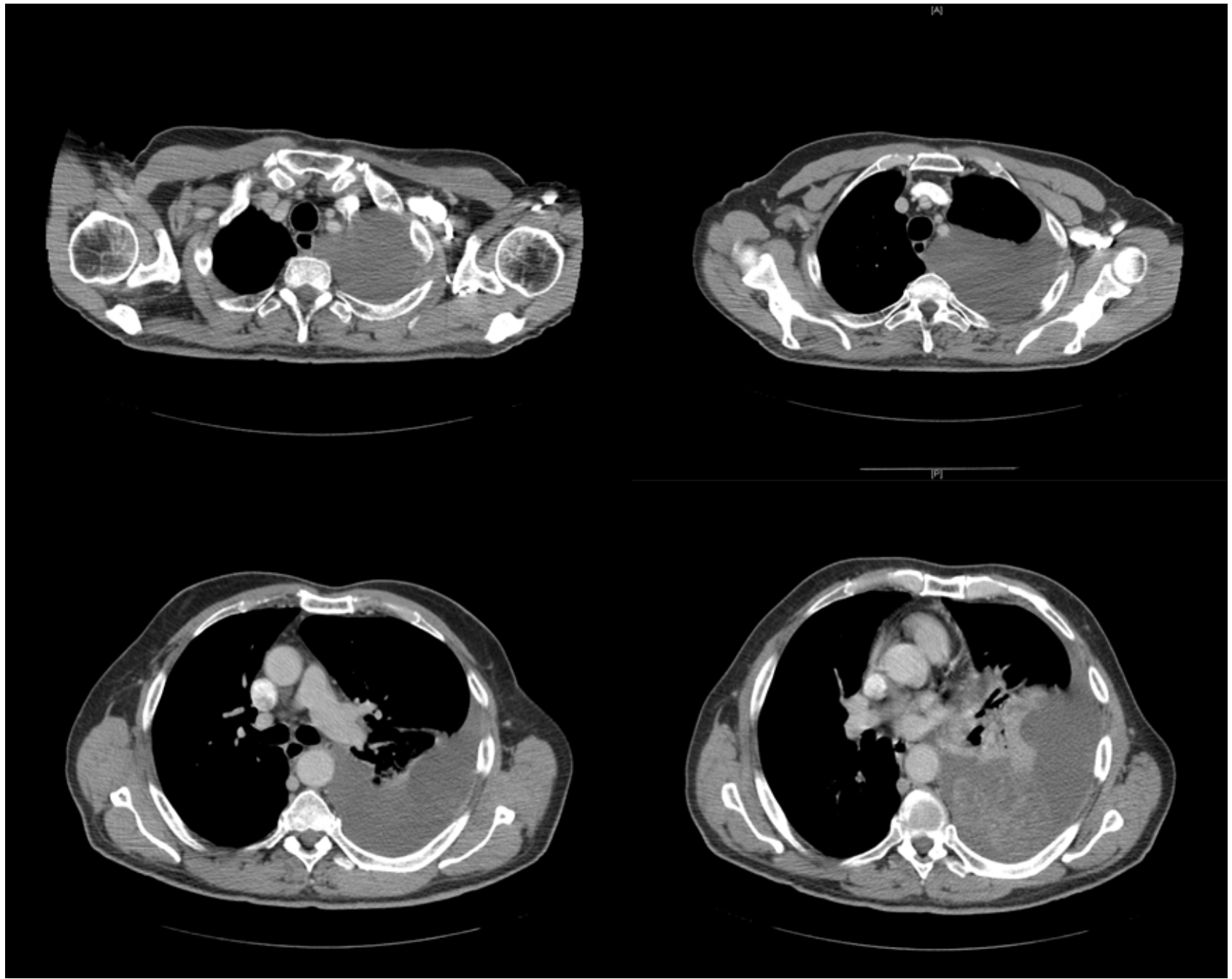

Figure 3: CT thorax showing a mass within the left pleural effusion. 


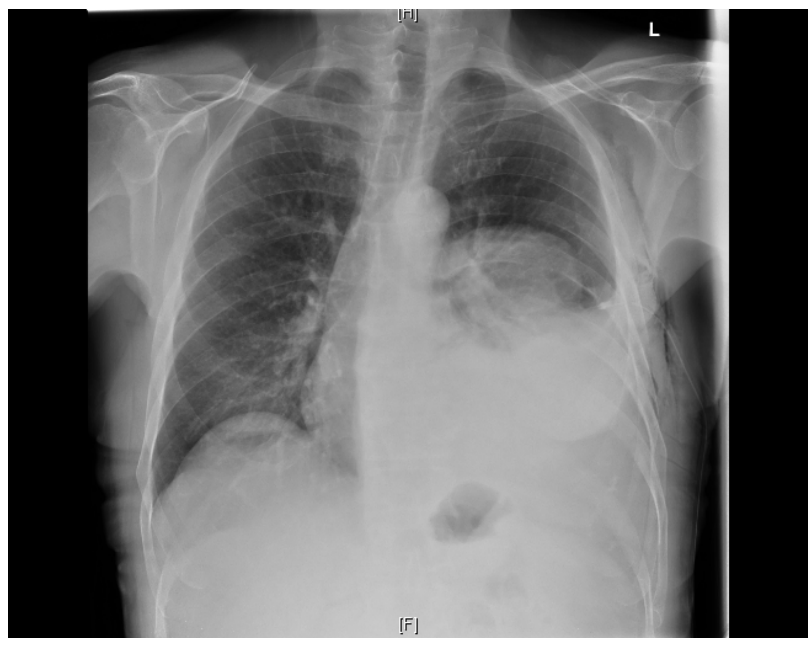

Figure 4: Left sided chest drain in situ. Left pleural effusion and mass in the left hemi-thorax.

Histology of core biopsies from the left pleural tumour showed a spindle-cell lesion with cells arranged around vascular spaces in a haemangio-pericytomatous pattern (Figure 5). The cells were positive for CD34, CD99 and bcl2-markers for a solitary fibrous pleural tumour. The presence of high mitotic activity, atypical mitotic figures and foci of necrosis favour the malignant variant of the tumour (Figure 6). On immunostaining for CK, SMA and Desmin, the tumour was negative. A diagnosis of malignant solitary fibrous tumour was made due to strong diffuse CD34 positivity.

During thoracotomy the pleural tumour was found to be diffuse and infiltrating the chest wall and lung and partial pleurectomy was performed.

The patient was started on imatinib $400 \mathrm{mg}$ daily but there was no tumour regression and after 4 months he succumbed to the disease.

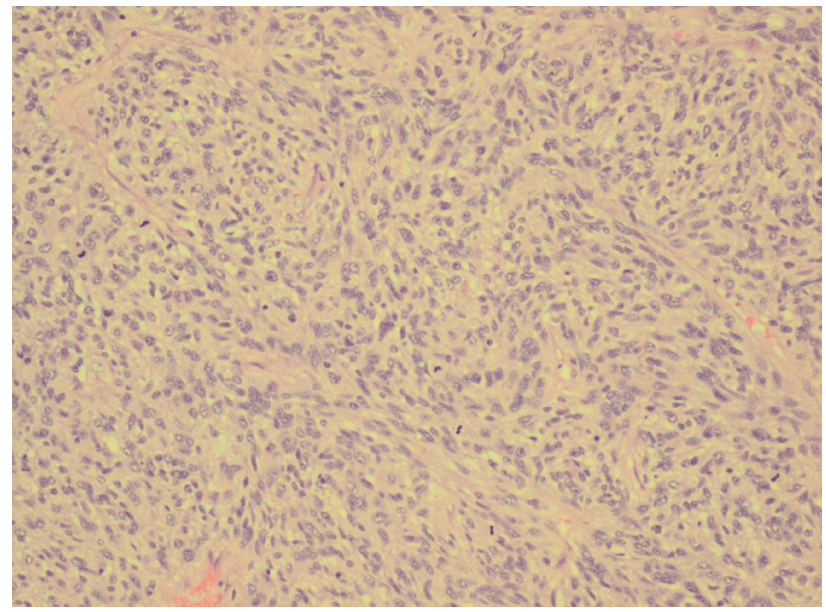

Figure 5: Malignant Solitary fibrous tumour (X10).

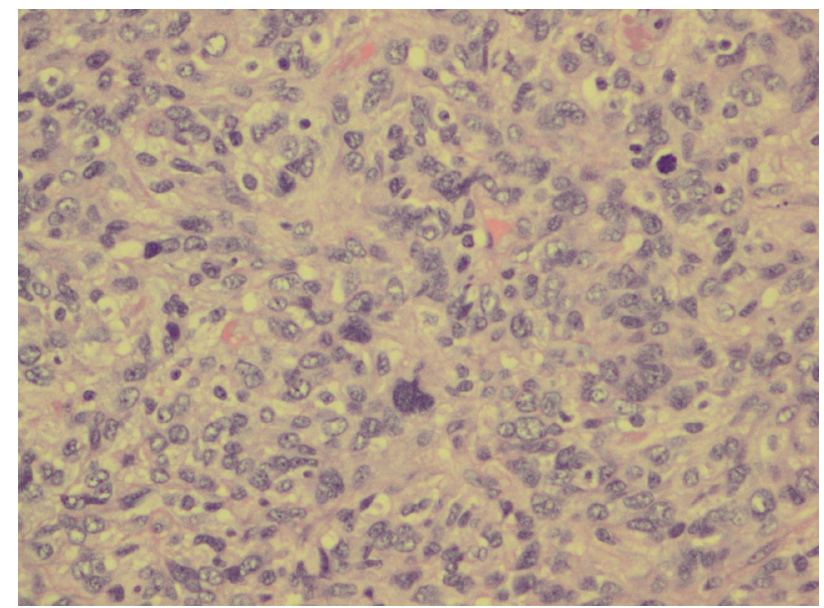

Figure 6: Mitosis and atypia in pleural tumour (X20).

\subsection{Case 2}

A 58-year-old gentleman presented to casualty with right shoulder pain and right-sided pleuritic chest pain associated with dyspnoea at rest especially when lying down. Patient denied haemoptysis but claimed he had decreased appetite and weight loss. He was hypertensive, ex-smoker (stopped 20 years previously) and worked as a street cleaner. The patient was treated for colonic adenocarcinoma (Duke B classification) with an anterior resection in 1987 and follow up colonoscopies were always negative to malignancy.

A CXR on presentation showed a right pleural effusion and a mass in the right lung base could not be excluded (Figure 7). A CT thorax showed small right subpleural effusion together with consolidation in the right lower and middle lobes (Figure 8).

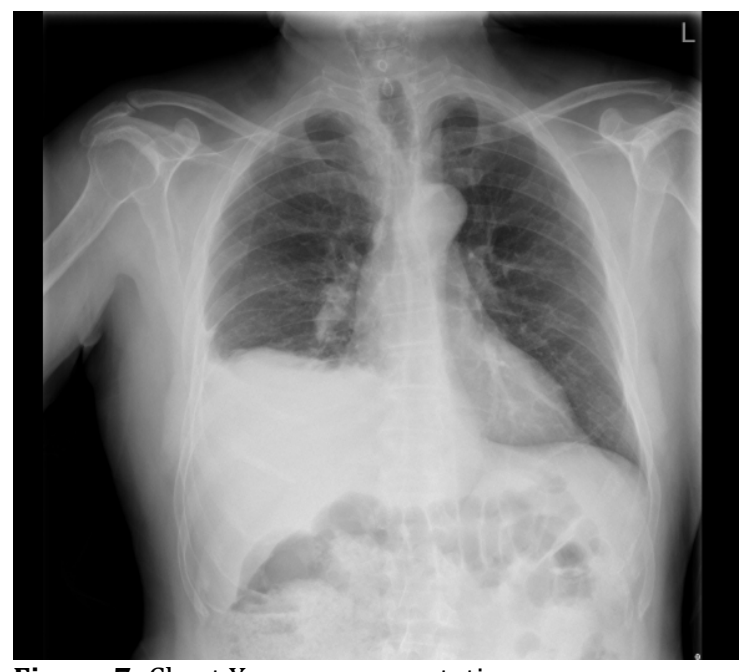

Figure 7: Chest X-ray on presentation. 


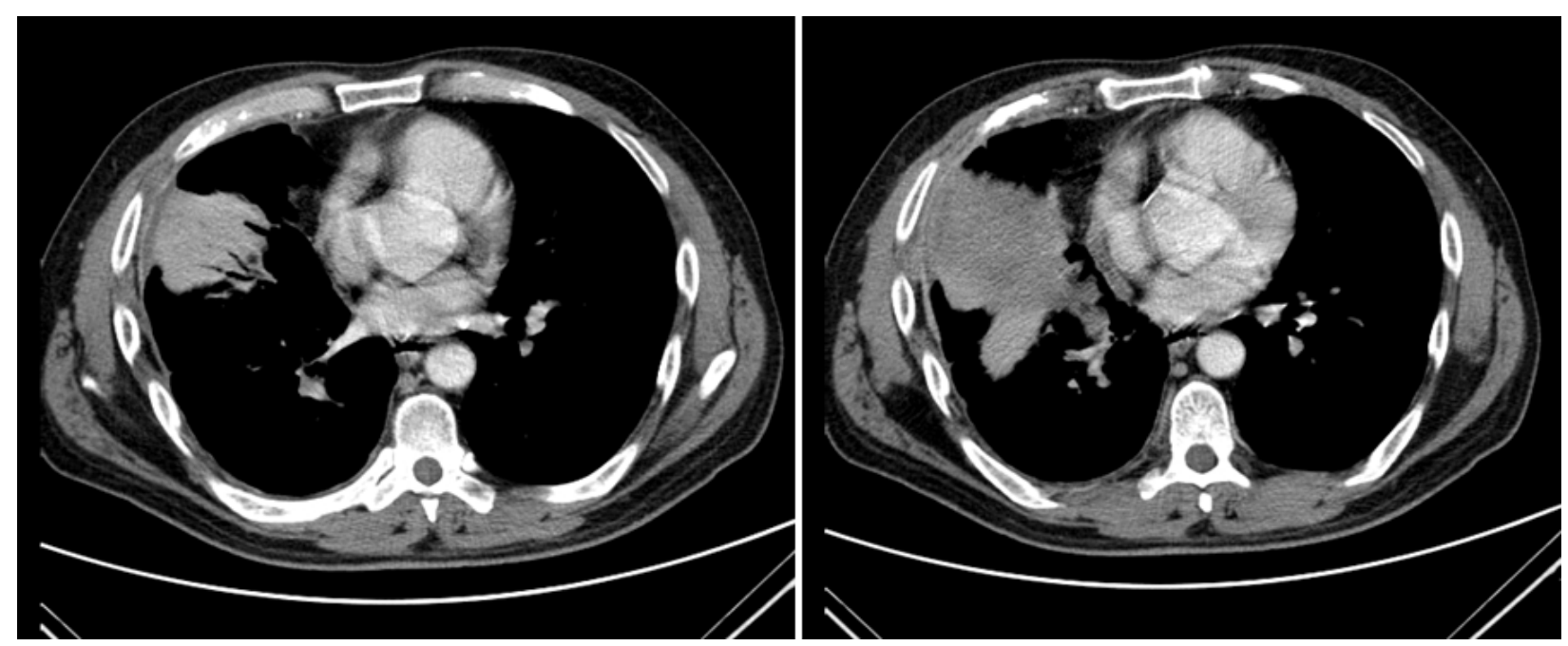

Figure 8: CT scan on presentation.

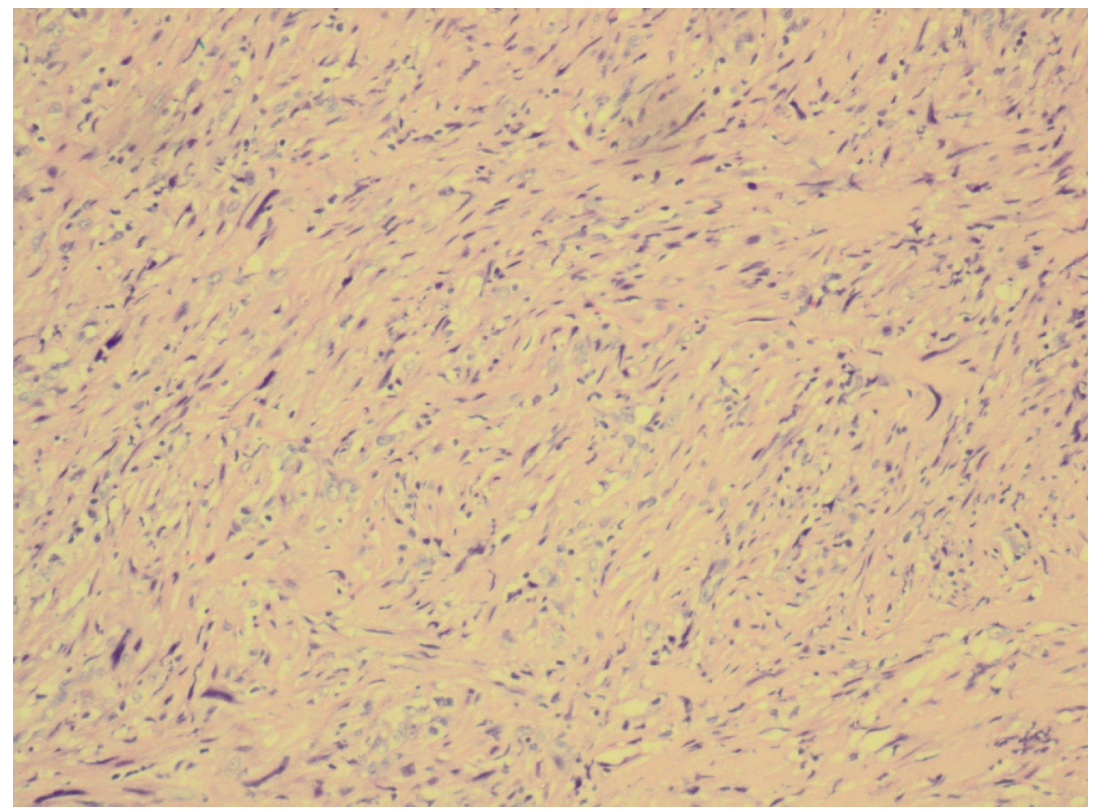

Figure 9: Mitosis and atypyia in spindle shaped cells with high level of mitosis (X10).

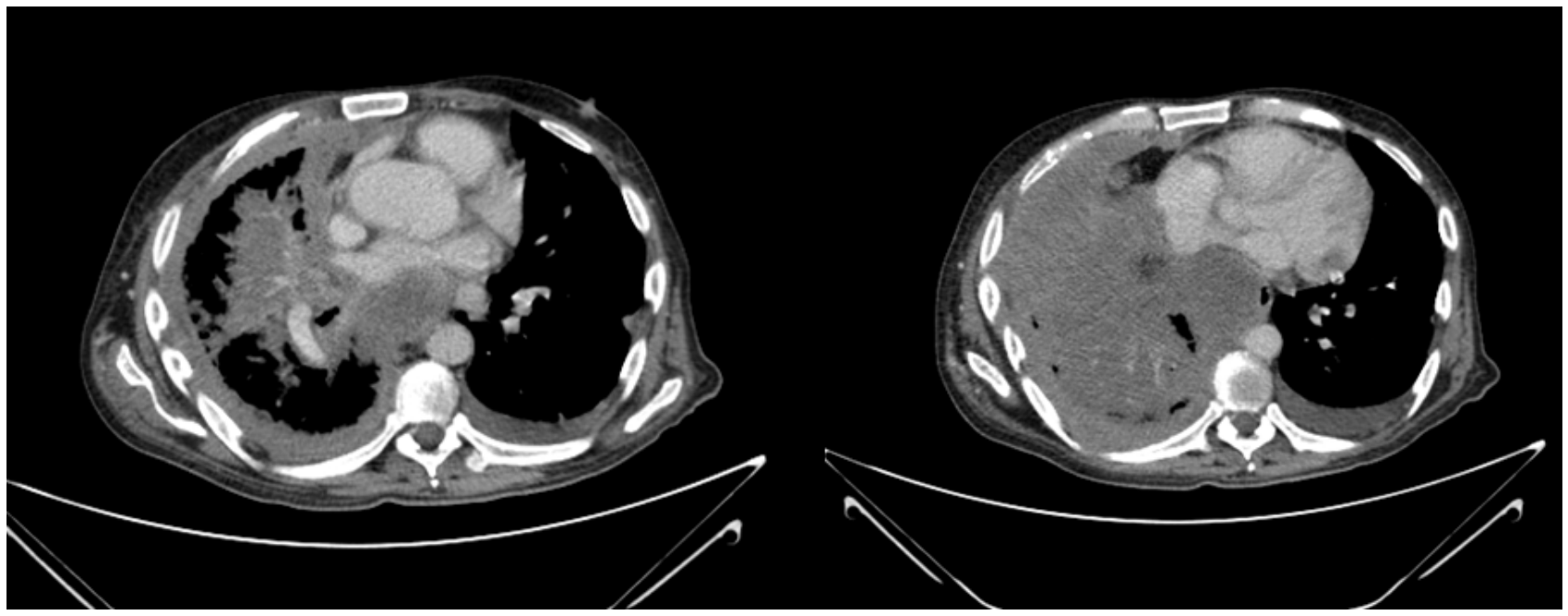

Figure 10: CT scan three months after presentation showing disease progression. 
The patient was referred for radical resection but it was unsuccessful due to extensive invasion of the tumour into both the thoracic wall and lung. Biopsies from the pleural mass represented a spindle cell neoplasm with increased cellularity, overlapping nuclei, cytological atypia, pleomorphism and high mitotic activity (6 per 10 HPF) (Figure 9). The tumour infiltrated fatty tissue in an invasive growth pattern. Minor foci of necrosis were seen. On immunohistochemistry, the cells were negative for mesothelial markers (CK, Calretinin, CK 5/6) as for the many other spindle cell markers (Desmin, S-100, CD99, B catenin, CD 34, CD117). Positively stained cells were present on SMA and BCL2 only. Melanoma markers/angiomyolipoma markers were also negative. The tumour showed positivity for p53 and Ki67 labelling index in approximately $80 \%$ of the cells. The morphology and the immunohistochemistry results of the tumour (CD34 negative, bcl-2 and SMA positive) were suggestive of a malignant solitary fibrous pleural tumour.

The patient was reviewed by the clinical oncologist who started him on imatinib $400 \mathrm{mg}$ daily with no regression of the tumour. CT thorax done three months after the first CT showed progression of the disease with increased right-sided pleural effusion (Figure 10). There were left lung metastasis and a smaller left-sided pleural effusion.

The patient continued to complain of increasing right-sided chest pain despite being started on a number of analgesics. A bone scan showed no bone metastasis. An elective right intercostal nerve block was done within two months of presentation. The patient passed away four and a half months from presentation.

In both patients imatinib was started without prior knowledge of the PDGFR expression status because the facility is not available in the country. Thus, the tumour specimens had to be sent abroad after both patients were started on this tyrosine kinase inhibitor. No mutations in PDGFR- $\alpha$ and PDGFR- $\beta$ and in c-KIT hot spots were detected in these two patients and both showed clinical and radiological disease progression in spite of imatinib treatment.

\section{Discussion and Conclusion}

We herein described two cases of a 67-year-old gentleman and a 58-year-old gentleman both diagnosed with a rare malignant tumour resident on an island inhabited by 420,000 people. Both tumours showed to be very aggressive in nature and were both inoperable at presentation.

Park et al. $^{7}$ concluded that not a single patient with advanced malignant solitary fibrous tumour showed complete or partial response to conventional chemotherapy although a large proportion remained with stable disease ${ }^{7}$. Tyrosine kinase inhibitors such as sunitinib have shown some promise in the treatment of SFTs. ${ }^{10}$ Also, a temozolomide-bevacizumab combination appear to have greater efficacy in treating SFTs than conventional chemotherapy such as doxorubicinifosfamide combinations. ${ }^{11}$

Imatinib mesylate works as a tyrosine kinase inhibitor targeting the PDGFR, the BCR-ABL, and c-KIT receptors. ${ }^{4}$ Imatinib is thought to be a potential treatment for malignant SFTs in tumour cells found to be positive for PDGFR- $\alpha$ and PDGFR- $\beta$, in the absence of c-KIT and PDGRFR mutations ${ }^{6}, 9$ Tyrosine kinase inhibitors might give beneficial effects in slowing disease progression in a number of patients, however, our patients only survived about 4 months after imatinib was started.

\section{Conflict of interest}

The authors declare that they have no conflicts of interest. The authors alone are responsible for the content and writing of the paper.

\section{Acknowledgement}

We would like to thank Dr. James DeGaetano, consultant histopathologist, Dr. Michelle Ceci, specialist trainee in pathology and their colleagues at the Histopathology Department, Mater Dei Hospital for their help with the preparation and interpretation of the histopathology slides. We would also like to acknowledge the contribution of Ms. Kimberley Grech, a medical student in the compilation of the data.

\section{References}

1. Santambrogio L, Nosotti M, Palleschi A, et al. Solitary fibrous tumor of the pleura presenting with syncope episodes when coughing. World J Surg Oncol. 2008;6:86.

2. Hanau CA, Miettinen M. Solitary fibrous tumour: histological and immunohistochemical spectrum of benign and malignant variants presenting at different sites. Hum Pathol. 1995; 26(4):440-9.

3. de Perrot M, Fischer S, Brundler MA, et al. Solitary fibrous tumours of the pleura. Ann Thorac Surg. 2002; 74(1):285-93.

4. Kim HJ, Lee HK, Seo JJ, et al. MR imaging of solitary fibrous tumours in the head and neck. Korean J Radiol 2005;6(3):136-42.

5. Robinson LA. Solitary fibrous tumour of the pleura. Cancer Control. 2006;13(4):264-9.

6. De Pas T, Toffalorio F, Colombo P, et al. Brief report: activity of imatinib in a patient with platelet-derived-growth-factor receptor 
positive malignant solitary fibrous tumour of the pleura. J Thorac Oncol. 2008;3(8):938-41.

7. Park MS, Ravi V, Conley A, et al. The role of chemotherapy in advanced solitary fibrous tumours; a retrospective analysis. Clin Sarcoma Res. 2013;3(1):7.

8. Constantinidou A, Jones RL, Scurr M, et al. Systemic therapy in solitary fibrous tumour. Proceedings of the $15^{\text {th }}$ Connective Tissue Oncologic Society Annual Meeting. Miami, Florida, USA; 2009.

9. Corless CL, Schroeder A, Griffith D, et al. PDGFRA mutations in gastrointestinal stromal tumours: frequency, spectrum and in vitro sensitivity to imatinib. J Clin Oncol. 2005;23(23):5357-64.

10. Stacchiotti $S$, Negri T, Palassini E, et al. Sunitinib malate and figitumumab in solitary fibrous tumor: patterns and molecular bases of tumor response. Mol Cancer Ther. 2010; 9(5):1286-97.

11. Park MS, Patel SR, Ludwig JA, et al. Activity of temozolomide and bevacizumab in the treatment of locally advanced, recurrent, and metastatic hemangiopericytoma and malignant solitary fibrous tumour. Cancer. 2011;117(21):4939-47. 